\title{
Patterns of local-regional failure after primary intensity modulated radiotherapy for nasopharyngeal carcinoma
}

Fangfang Kong ${ }^{1,2}$, Hongmei Ying ${ }^{1,2^{*}}$, Chengrun Du ${ }^{1,2}$, Shuang Huang ${ }^{1,2}$, Junjun Zhou ${ }^{1,2}$, Junchao Chen ${ }^{1,2}$, Lining Sun ${ }^{1,2}$, Xiaohui Chen ${ }^{1,2}$ and Chaosu Hu $u^{1,2}$

\begin{abstract}
Background: To analyze patterns of local-regional failure after primary intensity modulated radiotherapy (IMRT) for nasopharyngeal carcinoma (NPC).

Methods: A total of 370 non-metastatic NPC patients consecutively treated with IMRT (with or without chemotherapy) were analyzed. Radiotherapy was administered using a simultaneous integrated boost (SIB) technique at the total prescribed dose of 66-70.4Gy (2.0-2.2Gy per fraction). The location and extent of local-regional failures were transferred to the pretreatment planning computed tomography $(C T)$ for dosimetric analysis. The dose of radiation received by $V_{\text {recur }}$ (volume of recurrence) was calculated and analyzed with dose-volume histogram (DVH). Failures were classified as: "in field" if $95 \%$ of $V_{\text {recur }}$ was within the $95 \%$ isodose, "marginal" if $20 \%$ to $95 \%$ of $V_{\text {recur }}$ was within the $95 \%$ isodose, or "outside" if less than $20 \%$ of $V_{\text {recur }}$ was inside the $95 \%$ isodose.
\end{abstract}

Results: With a median follow up of 26 months, 25 local-regional failures were found in 18 patients. The 1- and 2-year actuarial local-regional control rates for all patients were $99.7 \%$ and $95.5 \%$ respectively. Among the 22 local-regional failures with available diagnostic images, 16 (64\%) occurred within the 95\% isodose lines and were considered in-field failures; 3 (12\%) were marginal and 3 (12\%) were outside-field failures.

Conclusions: Intensity-modulated radiotherapy provides excellent local-regional control for NPC. In-field failures are the main patterns for local-regional recurrence. Reducing the coverage of critical adjacent tissues in CTV purposefully for potential subclinical diseases was worth of study. Great attention in all IMRT steps is necessary to reduce potential causes of marginal failures. More studies about radioresistance are needed to reduce in-field failures.

Keywords: Intensity-modulated radiotherapy, Patterns of local-regional failure, Nasopharyngeal carcinoma

\section{Background}

Intensity-modulated radiotherapy (IMRT) is a major breakthrough in the treatment of NPC [1]. It is capable of improving dose conformity for complex tumor targets and better protection for the adjacent organs. Encouraging results with IMRT have been consistently reported [1-15]. Kwong [16] and Su [4] reported a 3- and 5- year local control rate of $100 \%$ and $97.7 \%$ for early-stage NPC treated with IMRT alone. For locally advanced NPC, 2and 5-year local control rate of 95.7\% [12] and 94.9\% [7] can be achieved after effective chemoradiotherapy.

\footnotetext{
*Correspondence: yinghongmei2013@163.com

'Department of Radiation Oncology, Fudan University Shanghai Cancer Center, 270 Dong'an Road, Shanghai 200032, P.R China

Department of Oncology, Shanghai Medical College, Fudan University, Shanghai 20032, P.R China
}

However, distant metastasis and post-treatment relapse remain as the main causes for NPC deaths. In 2000, Dawson et al. [17] firstly demonstrated that the majority $(75 \%)$ of local-regional relapses after IMRT as the primary treatment for head and neck cancer were in-field, in areas of previous disease which was judged to be at high risk at the time of RT planning. Their findings then motivate studies of dose escalation to the highest risk regions. Eleven years later, $\mathrm{Ng}$ et al. [1] reported similar failure patterns in NPC patients. Furthermore, they found that the locoregional failure rate was significantly related to the minimum target dose, and it was recommended to deliver at least 66.5Gy to the target volumes. A recent study by Orlandi et al. [18] showed that apart from the above factors, overall treatment time 
(OTT), patient positioning errors and anatomical changes during RT could also affect treatment results.

It is worthy of discussion because IMRT planning is usually associated with sharp dose gradients outside the target volumes; therefore, an inadequate definition of target volumes could increase the risk of geographic misses, which eventually lead to local-regional recurrence [18]. In addition, a case series reported by Cannon and Lee [19] showed that the risk of marginal miss may be increased when excessive parotid gland sparing was pursued.

The aim of this study is to analyze the local-regional failure patterns following IMRT for NPC in our institution. This analysis allows us to assess the adequacy and the overall quality of the treatment technique.

\section{Methods}

\section{Patients and pretreatment evaluations}

From December 2007 to April 2012, 370 newly diagnosed non-metastatic NPC patients treated by definitive IMRT in Shanghai Cancer Center of Fudan University were enrolled in this study. All patients underwent disease restaging using the AJCC 2010 staging system. This study was approved by the Institutional Review Boards of Fudan University Shanghai Cancer Center. Written informed consent was obtained from the patient for the publication of this report and any accompanying images.

\section{Intensity-modulate radiotherapy Immobilization and simulation}

Patients were immobilized in the supine position with a thermoplastic head and shoulder mask. Intravenous contrast-enhanced CT using slice thickness of $5 \mathrm{~mm}$ was performed for planning. Image fusion of the T1 sequences with gadolinium enhanced MRI was performed with the CT simulation images for target delineation. The CT data were imported to treatment planning system for treatment design.

\section{Target delineation}

The target volumes were defined in accordance with the International Commission on Radiation Units and Measurements Reports 50 and 62 [20,21]. The primary gross tumor volume (GTV_P) and involved lymph nodes $\left(G T V \_N\right)$ included all gross tumors was determined by imaging, clinical, and endoscopic findings. The enlarged retropharyngeal nodes were outlined together with primary GTV, as the GTV_P on the IMRT plans. For patients who received neoadjuvant chemotherapy, the prechemotherapy volume of the primary lesion was used for GTV-P delineation, and the post-chemotherapy volume of the lymph nodes was used for GTV-N delineation.

The clinical target volume (CTV) was defined as the GTV plus 5- to 10-mm margin to encompass any microscopic extension, together with the regional lymphatics.
Two clinical target volumes (CTVs) were defined in our radiotherapy: CTV1 and CTV2. The CTV1 was defined as the high-risk region that included GTV_P plus 5- to 10-mm margin; CTV1 should also encompass the entire nasopharynx, skull base, parapharyngeal space, retropharyngeal lymph nodal regions, the anterior second of the clivus, inferior sphenoid sinus, pterygoid fossae, the posterior third of the nasal cavity and maxillary sinuses, and any high risk nodal regions, including the bilateral upper deep jugular nodes, and the near station of the positive lymph nodes. Neck levels IB (submandibular nodes) were selectively irradiated only if there was extensive nodal disease on the ipsilateral IIA/IIB region, extracapsular extension of the IIA lymph nodes, or tonsilla and lingual root involved by the primary tumor. The levels IA (submental nodes) were delineated only if the submandibular nodes or oral cavity were grossly involved by disease. The low-risk CTV (CTV2) referred to levels IV and $\mathrm{Vb}$ without metastatic cervical lymph nodes. There were two corresponding PTV_Cs in our radiotherapy: PTV_C1 (CTV1 $+3 \mathrm{~mm})$ and PTV_C2 (CTV2 +3 mm). The PTV_Cs would encompass the corresponding CTV with a $3-\mathrm{mm}$ margin in all directions. However, when the CTV was near critical organs, such as the brainstem, spinal cord, PTV_C was generated as small as $1 \mathrm{~mm}$.

The organs at risk (OAR) include the spinal cord, brain stem, optic chiasm, optic nerves, eyeballs, lens, temporal lobes, parotid glands, oral mucosa, larynx and temporomandibular joints. A 5-mm margin was added to the spinal cord and brainstem during optimization to form the planning organ-at-risk volume (PRV).

\section{Treatment planning and delivery}

All patients were treated with external-beam radiation therapy using 6-MV photons, 7-9 radiation fields. The treatment technique was simultaneous integrated boost (SIB) technique. The dose prescribed to PTV-G (GTV $+5 \mathrm{~mm}$ ) was $66 \mathrm{~Gy}$ in 30 fractions for T1-T2 disease, 70.4Gy in 32 fractions for T3-T4 disease, and 66Gy in 30 or 32 fractions for lymph nodes involved by disease. The dose delivered to PTV-C1and PTV-C2 was 60Gy and 54Gy, respectively. All patients were treated one fraction per day, 5 days per week.

\section{Chemotherapy}

About $92.2 \%$ patients received cisplatin based chemotherapy including neoadjuvant chemotherapy, concurrent chemotherapy and adjuvant chemotherapy. The most common regimen of neoadjuvant and adjuvant chemotherapy included two to three cycles of TP (docetaxel $60 \mathrm{mg} / \mathrm{m}^{2} /$ day, day 1 , cisplatin $25 \mathrm{mg} / \mathrm{m}^{2} /$ day, days 1-3), TPF (docetaxel $60 \mathrm{mg} / \mathrm{m}^{2} /$ day, day 1 , cisplatin $25 \mathrm{mg} / \mathrm{m}^{2} /$ day, days $1-3$, and 5 -fluorouracil $0.5 \mathrm{~g} / \mathrm{m}^{2}$ 
/day, days $1-3$ ), or GP (gemcitabine $1 \mathrm{~g} / \mathrm{m}^{2} /$ day, day 1 , day 8 , cisplatin $25 \mathrm{mg} / \mathrm{m}^{2} /$ day, days $1-3$ ) regimen. Induction chemotherapy was given every 3 weeks. Four weeks after the completion of RT, the adjuvant chemotherapy was administered every 3 weeks. Concurrent chemotherapy consisting of $80 \mathrm{mg} / \mathrm{m}^{2}$, days $1-3$ of cisplatin, every 3 weeks for 2 to 3 cycles.

\section{Patient evaluation}

All patients were evaluated weekly for treatment response and toxicity during radiation therapy. After IMRT, patients were clinically evaluated at predefined intervals, typically every 3 months in the first 2 years, every 6 months from the third year to the fifth year, and annually thereafter. Each follow-up included indirect mirror examination for the nasopharynx and palpation of neck nodes. MRI of the nasopharynx, chest CT scan, and ultrasound of abdomen were performed 3 months after the completion of IMRT and every 6-12 months thereafter. Additional tests were ordered when indicated to evaluate local or distant relapse.

\section{Definition of failure site}

For patients with local-regional failure, the recurrent tumor volume $\left(\mathrm{V}_{\text {recur }}\right)$ was identified on MRI scans or CT scans obtained at the time when recurrence was diagnosed and transferred to the pretreatment planning CT. The exact site and extent of each tumor were then compared with the pretreatment planning CT data sets, focusing on the $95 \%$ isodose lines. The dose of radiation received by $\mathrm{V}_{\text {recur }}$ was calculated and analyzed with dose-volume histogram (DVH). The failures were categorized as occurring inside or outside the high dose target volume, depending on the location of $V_{\text {recur: "in }}$ field" if $95 \%$ of $V_{\text {recur }}$ was within the $95 \%$ isodose, "marginal" if $20 \%$ to $95 \%$ of $\mathrm{V}_{\text {recur }}$ was within the $95 \%$ isodose, or "outside" if less than $20 \%$ of $\mathrm{V}_{\text {recur }}$ was inside the $95 \%$ isodose [17].

\section{Statistical methods}

The follow-up period was measured from the first day of treatment. The Statistical Package for Social Sciences (SPSS version 16.0) software was used for statistical analysis. Kaplan-Meier method was used to calculate the cumulative local failure-free survival (LFFS), regional failure- free survival (RFFS) and overall survival (OS).

\section{Results}

\section{Rates of local-regional recurrence}

A total of 370 patients were analyzed. Characteristics of patients, tumor stage and treatment factors were detailed in Table 1. The median follow-up was 26 months, with a range from 3 months to 62 months. The 1- and 2-year actuarial local-regional control rates for all patients were
Table 1 Patient characteristics and treatment factors ( $\mathbf{n}=\mathbf{3 7 0}$ )

\begin{tabular}{ll}
\hline Characteristic & Patients (\%) \\
\hline Gender & \\
Male & $273(73.8)$ \\
Female & $97(26.2)$ \\
Age (yr) & \\
Median & 50 \\
Range & $9-79$ \\
WHO histologic type & \\
I & $3(0.8)$ \\
॥ & $76(20.5)$ \\
III & $285(77)$ \\
Others & \\
Tumor classification & $6(1.7)$ \\
T1-2 & \\
T3-4 & $198(53.5)$ \\
\hline
\end{tabular}

Node classification

No-1

$139(37.5)$

$\mathrm{N} 2-3$

$231(62.5)$

Stage

I/II

$69(17.2)$

III/IV

$301(82.8)$

Treatment factors

IMRT treatment duration (days)

Median (range)

$45(40-58)$

RT alone

$29(7.8)$

Chemotherapy

$341(92.2)$

WHO World Health Organization, RT radiotherapy.

${ }^{a}$ Other WHO histologic type including nasopharyngeal adenoid cystic carcinoma, poorly differentiated adenocarcinoma, small cell carcinoma and carcinomatous change.

99.7\% (95\% confidence interval [CI]: 100-99.1\%) and 95.5\% (95\% CI: 93-98\%) respectively. The 2-year LFFS, RFFS and OS were 97.7\%, 97.0\% and 94.1\%, respectively.

At their last follow-up visit, 18 patients (4.9\%) developed clinical or radiographic local-regional recurrences. $78 \%(14 / 18)$ of the patients were locally advanced (staged T3 or T4). The median time from treatment to local-regional recurrence was 20.5 months (range 11 to 41 months). Twelve patients with a local-regional recurrence were successfully salvaged with surgery alone (4), chemotherapy alone (4), RT alone (1), neck dissection and RT (1), or RT and chemotherapy (2). The ultimate crude local regional control rate was $66.7 \%$. For the six patients with local-regional recurrences that could not be salvaged, four patients suffered distant metastasis before, simultaneously, or shortly after local-regional recurrence and the other two refused to accept any treatment. 


\section{Dosimetric data}

Table 2 shows the DVH statistics for patients of localregional recurrence. On average, the target volumes had excellent coverage and only $1.7 \%$ of the GTV_P and $0.3 \%$ of the GTV_N received $<95 \%$ of the prescribed dose. The majority (91\%-96\%) of the GTV_P and GTV_N actually received more than $100 \%$ of the prescribed dose. A similar situation was found in CTV1 and CTV2. The mean dose to the CTV1 was 65.9Gy and 56.7Gy to the CTV2. Volume (\%) receiving less than $95 \%$ of the prescribed dose was $0.7 \%$ to CTV1 and $0.4 \%$ to CTV2.

\section{Patterns of failure}

In the 18 patients who developed into local-regional recurrences, 7 patients had isolated regional recurrences, other 7 patients had isolated local recurrences, and 4 patients had both local and regional recurrences. 5 patients had simultaneous distant metastasis, including lung and bone metastasis.

A total of 25 recurrences were observed in the above 18 patients, 16 (64\%) occurred within the 95\% isodose lines and were considered in-field failures; $3(12 \%)$ were marginal, occurring in a steep dose gradient region at the margin of the high-dose PTV-C, the other 3 (12\%) were outside-field failures. There were 3 missing values because of the unavailability of the diagnostic image. The sites of local-regional recurrences are detailed in Table 3.

As show in Table 3, the average minimum, mean and maximum dose delivered to $V_{\text {recur }}$ for marginal recurrence were 28Gy, 61.8Gy and 72.6Gy, respectively. Regarding the in-field recurrences, the average minimum dose to $\mathrm{V}_{\text {recur was }}$ 59.3Gy (range 37.6Gy to 69.1Gy). The average mean dose of radiation to the in-field recurrences was 69.3Gy (range 65.1Gy to 73.5Gy). The average maximum dose of radiation to the in-field recurrences was 73.5Gy (range 69.3Gy to 77.3Gy). Figure 1 demonstrates axial dose distributions through the epicenter of the recurrent volumes occurring in-field, marginal and outside-field.

\section{Discussion}

There is little controversy that IMRT is the treatment of choice for NPC because dosimetric studies showed clear advantages by improving dose conformity for complex tumor targets and better protection of the adjacent organs [1]. The 1- and 2-year local-regional control rates of $99.7 \%$ and $95.5 \%$ in the present study are excellent and similar to reports from other centers [1-15].

As shown in Table 3, most of the relapses in our study occurred in locally advanced disease, within or marginally to the high dose region. Three out-field failures were observed in this study. Patient 7 (staged T3N3b) had treatment failure within a spared parotid gland. Parotid node involvement occurs in only $1 \%$ of cases [22]. This low proportion of patients does not justify the inclusion of this level in local-regional CTV. However, Cannon et al. [19] suggested that for patients who had multilevel nodal disease, including disease in level II, and underwent a neck dissection that could have contributed to modification of lymphatic drainage, parotid nodes could be considered for inclusion in local-regional CTV. Patient 14 (staged T2N1) suffered 3 times of regional recurrences one after another, two of them occurred in submandibular nodes. Neck levels IB (submandibular nodes) were selectively irradiated only if there was extensive nodal disease on the ipsilateral IIA/IIB region, extracapsular extension of the IIA lymph nodes, or tonsilla and lingual root involved by the primary tumor in our institution. The recent meta-analysis by Ho et al. reported that IB node involvement occured in only $3 \%$ of cases [22]. This low risk of involvement also supports the elective treatment of level IB. The $3^{\text {rd }}$ relapse occurred in neck levels IIA which grew alongside the space between the muscles, and then it was defined as "outside-field" failures. The potential reason is the neck dissections the patient subjected to after the previous relapses, which could have modified his lymphatic drainage.

It is generally recommended that a total dose of 70Gy with conventional fractionation should be given, but it is

Table 2 Dose-volume histograms (DVHs) statistics for patients of local-regional recurrence

\begin{tabular}{lllll}
\hline & GTV_P & GTV_N & CTV1 & CTV2 \\
& Average (range) & Average (range) & Average (range) & Average (range) \\
\hline Volume (cc) & $88.5(35-140.3)$ & $85(34.2-167)$ & $298.6(117.8-530.8)$ & $124(27.1-228.1)$ \\
Dmax (Gy) & $75.5(71.7-77.6)$ & $73.2(71.2-75.3)$ & $75.2(70.4-77.6)$ & $61.7(58.9-63.9)$ \\
Dmean (Gy) & $71.5(68-72.9)$ & $68.7(68-69.9)$ & $65.9(62.1-69.2)$ & $56.7(55.5-57.5)$ \\
Dmin (Gy) & $59.9(52.7-65.4)$ & $58.8(48.4-64.2)$ & $43.4(17.5-54.5)$ & $43.3(7-51)$ \\
V95\% & $1.7(0-6.3)$ & $0.3(0-1.5)$ & $0.7(0.2-2.1)$ & $0.4(0-1.4)$ \\
V100\% & $91.3(77.3-98.9)$ & $95.6(90.9-98.9)$ & $96.5(92.1-99.1)$ & $96.6(93.9-99.5)$ \\
V110\% & $0.06(0-1.1)$ & $0.7(0-3.8)$ & $44(2.1-76)$ & $5.7(0-14.5)$ \\
\hline
\end{tabular}

GTV_P gross tumor volume of primary tumor, GTV_N gross tumor volume of involved lymph nodes, CTV1 clinical tumor volume of the high-risk region, CTV2 clinical tumor volume of lymph nodal regions at low risk. Dmax $=$ Maximum dose, Dmean $=$ Mean dose, Dmin $=$ Minimum dose. V95\% $=\%$ volume receiving $<95 \%$ of the prescribed dose, $\mathrm{V} 100 \%=\%$ volume receiving $>100 \%$ of the prescribed dose, $\mathrm{V} 110 \%=\%$ volume receiving $>110 \%$ of the prescribed dose. 
Table 3 Details of recurrent patients and their local-regional failures

\begin{tabular}{|c|c|c|c|c|c|c|c|c|c|}
\hline \multirow[b]{2}{*}{ No. } & \multirow[b]{2}{*}{ Stage } & \multirow[b]{2}{*}{$\begin{array}{l}\text { Site of } \\
\text { relapse }\end{array}$} & \multirow[b]{2}{*}{$\begin{array}{l}\text { Location of the } \\
\text { recurrence volume }\end{array}$} & \multicolumn{5}{|c|}{ Dose-volume histograms statistics to recurrence volume } & \multirow[b]{2}{*}{$\begin{array}{l}\text { Type of } \\
\text { relapse }^{a}\end{array}$} \\
\hline & & & & $\begin{array}{l}\text { Vrecur } \\
\text { (cc) }\end{array}$ & $\begin{array}{l}\text { Dmean } \\
\text { (Gy) }\end{array}$ & $\begin{array}{l}\text { Dmin } \\
\text { (Gy) }\end{array}$ & $\begin{array}{l}\text { Dmax } \\
\text { (Gy) }\end{array}$ & $\begin{array}{l}V_{95 \%} \\
(\%)\end{array}$ & \\
\hline \multirow[t]{2}{*}{1} & T4N3b & Local & CTV & 13.9 & 69.9 & 48.5 & 75.2 & 97.9 & In-field \\
\hline & & Regional & CTV & 2.7 & 68.9 & 64.6 & 71.3 & 100 & In-field \\
\hline 2 & T4N1 & Local & Marginal to CTV & 10.5 & 70 & 51.5 & 77 & 94.3 & marginal \\
\hline 3 & T4NO & Local & CTV & 32.9 & 70.9 & 37.6 & 75.4 & 97.7 & In-field \\
\hline \multirow[t]{2}{*}{4} & T3N3b & Local & Marginal to CTV & 13.9 & 62.1 & 11.8 & 74.1 & 74.7 & marginal \\
\hline & & Regional & CTV & 3.6 & 67.5 & 63.9 & 71.9 & 100 & In-field \\
\hline 5 & $\mathrm{~T} 2 \mathrm{~N} 1$ & Local & CTV & 15.1 & 67.6 & 60 & 71.2 & 100 & In-field \\
\hline 6 & T1N0 & Local & CTV & 10.2 & 65.1 & 53.3 & 71.7 & 100 & In-field \\
\hline \multirow[t]{2}{*}{7} & T3N3b & Local & CTV & 2.2 & 70 & 63 & 76.8 & 100 & In-field \\
\hline & & Regional & Outside CTV & 8.4 & 14.2 & 11.2 & 37.7 & - & outside \\
\hline \multirow[t]{2}{*}{8} & T3N1 & Local & GTV & 10.6 & 73.5 & 69.1 & 76.3 & 100 & In-field \\
\hline & & Regional & CTV & 5.5 & 69.6 & 65.9 & 73.6 & 100 & In-field \\
\hline 9 & T4NO & Local & GTV & 9.3 & 72 & 61.8 & 74 & 99.2 & In-field \\
\hline 10 & T3N2 & Local & CTV & 15.2 & 71.9 & 63.3 & 77.3 & 100 & In-field \\
\hline 11 & T4N1 & Local & CTV & 8 & 72.1 & 66 & 73.8 & 100 & In-field \\
\hline 12 & T4N3b & Regional & - & - & - & - & - & - & - \\
\hline 13 & T3N2 & Regional & CTV & 20.6 & 67.8 & 51.2 & 72.6 & 99.6 & In-field \\
\hline \multirow[t]{3}{*}{14} & $\mathrm{~T} 2 \mathrm{~N} 1$ & Regional 1 & Outside CTV & 3.6 & 45.3 & 34.6 & 56.7 & - & outside \\
\hline & & Regional 2 & Marginal to CTV & 12.6 & 53.4 & 20.6 & 66.7 & 32.5 & marginal \\
\hline & & Regional 3 & Outside CTV & 2.7 & 54.2 & 46.6 & 61.4 & 19.1 & outside \\
\hline 15 & T3N2 & Regional & - & - & - & - & - & - & - \\
\hline 16 & T3N3b & Regional & CTV & 4.2 & 66.5 & 61.3 & 69.3 & 100 & In-field \\
\hline 17 & $\mathrm{~T} 1 \mathrm{~N} 2$ & Regional & GTV & 2.2 & 70.1 & 67.9 & 72.4 & 100 & In-field \\
\hline \multirow[t]{2}{*}{18} & T3N3b & Regional 1 & - & - & - & - & - & - & - \\
\hline & & Regional 2 & CTV & 22.2 & 65.6 & 51.4 & 73 & 98.8 & In-field \\
\hline
\end{tabular}

CTV clinical target volume, GTV gross tumor volume; V95\%=\% of volume of failure receiving at least $95 \%$ of prescribed total dose, Vrecur the recurrent tumor volume

${ }^{a}$ In-field refers to $95 \%$ of the recurrence volume receiving more than $95 \%$ of the prescribed dose. Marginal refers to $20-95 \%$ of the recurrence volume receiving $95 \%$ of the prescribed dose. Outside refers to less than $20 \%$ of the the recurrence volume receiving $95 \%$ of the prescribed dose.

difficult to be administered without severe side effects in advanced T4 disease, even by IMRT [18]. Ng et al. [1] suggested a possible strategy to lessen the dose constraint criteria of selected neurologic structures (i.e. to sacrifice one side of the optic nerve or temporal lobe). In our study, patient 2 (stage T4N1) and patient 4 (stage T3N3b) developed cavernous sinus and orbital apex recurrence, which were relatively high locations in NPC. The possible reasons include missing of subclinical target volume and compromise with OAR. This also suggests the importance of cooperation between clinicians and radiologists. And great attention should be paid to the treatment precision for patients with adequate but very tight dose coverage to the target volumes, especially for patients with the very steep dose gradient proximal to the critical neurologic structures.

As show in our study, the main patterns of localregional recurrence are in-field failures. A recent Medline review by Hong B et al. [23] showed that radioresistance may be the ultimate cause of a local-regional failure. They reviewed articles published on clinical and preclinical studies targeting tumor hypoxia and found that tumor hypoxia was common in NPC; it was associated with disease progression and resistance to therapy. In our series, $64 \%$ recurrences were located well within the $95 \%$ dose region. In these cases, it is reasonable to assume that there are nonuniform clonogenic cell density and radiosensitivity within the same target. Hence, we hope that with the development of radiation biology, a smarter, nonuniformly increased dose distribution can be established to reduce in-field failure as much as possible.

Despite of the prevailing use of IMRT in the treatment of NPC, optimal target volumes especially the clinical target volumes (CTVs) have not been sufficiently addressed. Our results suggest that the definition of CTVs currently 


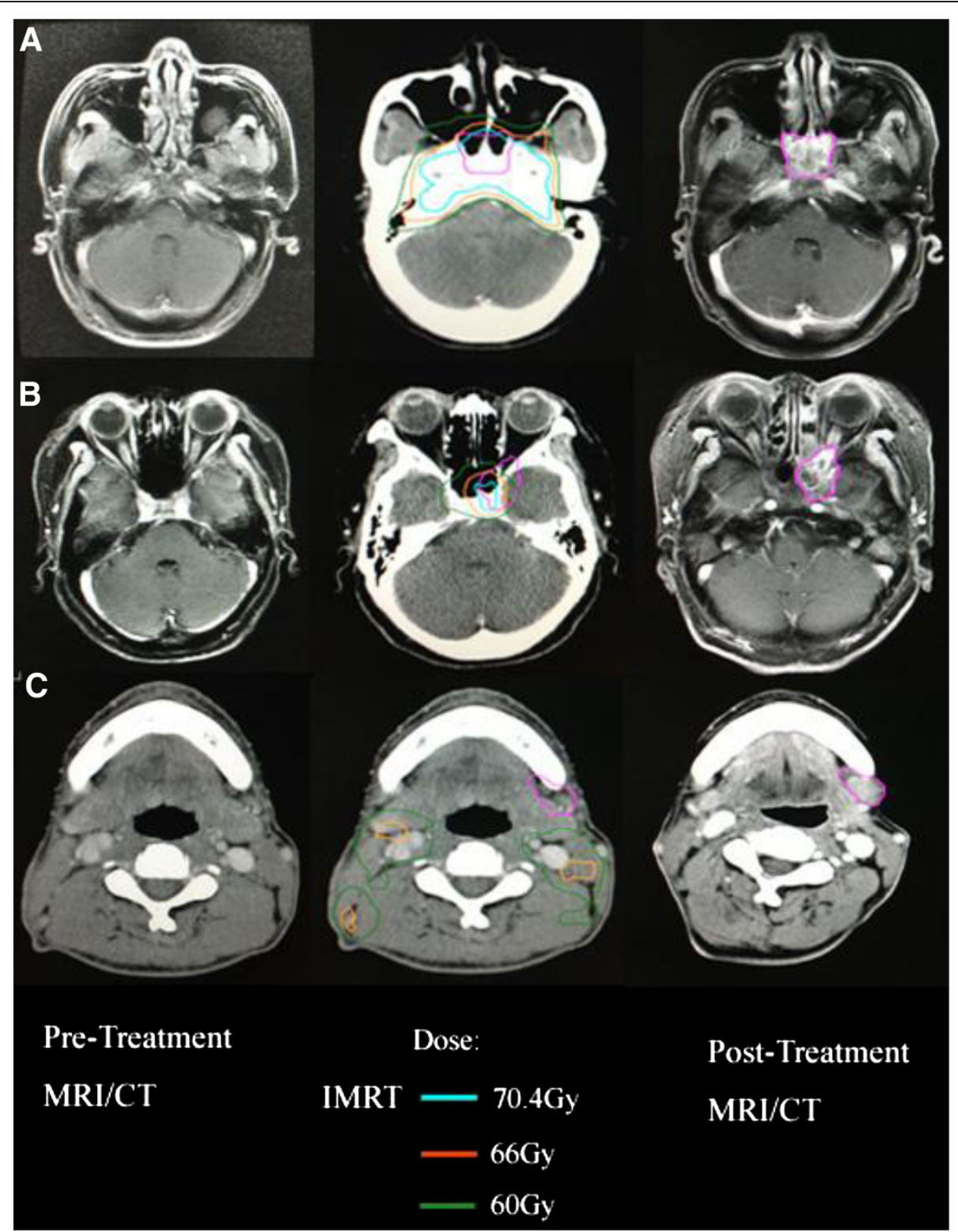

Figure 1 Disease extent for patients having local-regional failures. A, In-field failure. B, Marginal failure. C, Outside-field failure. Left, Pretreatment magnetic resonance imaging $(M R I / C T)$. Middle, The recurrent tumor volumes were transferred from the diagnostic MRI/CT at the time of recurrence to the planning computed tomography to show doses delivered to the recurrence sites. Right, MRI/CT at time of failure. IMRT = intensity-modulated radiotherapy.

used in our institution is sufficient. It provided excellent control in both the primary disease and involved neck areas. As reducing high-dose radiation to normal critical tissues is one of the major purposes of IMRT, it is questionable that if we can purposefully reduce the coverage of critical adjacent tissues in CTV for potential subclinical diseases. Lin et al. [24] found that IMRT using a reducedvolume technique did not increase incidence of local and /or regional recurrence that could be attributed to the reduction of clinical target volume adjacent to the primary disease. But further optimization and prospective researches are needed.
There were several limitations of this study. First, since the interval for enrollment was about 5 years, various factors such as radiation techniques, radiation doses and chemotherapy regimens have evolved. Second, due to the relatively small sample size and short time follow-up, the current findings could only be taken as preliminary. In order to illustrate patterns of local-regional failure and possible reasons following primary IMRT for NPC, longer follow-up and a large sample of uniform treatment are needed for further research.

In conclusion, our data shows excellent local-regional control by IMRT for NPC. The 1- and 2-year actuarial 
local-regional control rates for all patients were $99.7 \%$ and $95.5 \%$ respectively. In-field failures (64\%) are the main patterns for local-regional recurrence. The occurrence of marginal failures implies that great attention should be paid in all IMRT steps to reduce potential causes of marginal failures. And studies focusing on radioresistant should be pursued to reduce in-field failures in the future. Reducing the coverage of critical adjacent tissues in CTV purposefully for potential subclinical diseases was worth of study.

\section{Abbreviations}

IMRT: Intensity-modulated radiotherapy; NPC: Nasopharyngeal carcinoma; RLN: Retropharyngeal lymph node; DVH: Dose-volume histogram.

\section{Competing interests}

The authors declare that they have no competing interests.

\section{Authors' contributions}

$\mathrm{CD}, \mathrm{SH}$ and $\mathrm{HY}$ participated in the treatment panning, contributed to the data collection. $\mathrm{HY}, \mathrm{CH}$ and FK participated in its design and coordination. $J Z$ and $X C$ conceived of the study and participated in the data collection. $\mathrm{FK}$ and $\mathrm{HY}$ performed the statistical analysis, and drafted the manuscript. All authors read and approved the final manuscript.

Received: 2 August 2013 Accepted: 16 February 2014 Published: 19 February 2014

\section{References}

1. Ng WT, Lee MC, Hung WM, Choi CW, Lee KC, Chan OS, Lee AW: Clinical outcomes and patterns of failure after intensity-modulated radiotherapy for nasopharyngeal carcinoma. Int J Radiat Oncol Biol Phys 2011, 79(2):420-428.

2. Cao CN, Luo JW, Gao L, Yi JL, Huang XD, Wang K, Zhang SP, Qu Y, Li SY, Cai WM, Xiao JP, Zhang Z, Xu GZ: Clinical outcomes and patterns of failure after intensity-modulated radiotherapy for T4 nasopharyngeal carcinoma. Oral Oncol 2013, 49(2):175-181.

3. Wang R, Wu F, Lu H, Wei B, Feng G, Li G, Liu M, Yan H, Zhu J, Zhang Y, Hu $K$ : Definitive intensity-modulated radiation therapy for nasopharyngeal carcinoma: long-term outcome of a multicenter prospective study. J Cancer Res Clin Oncol 2013, 139(1):139-145.

4. Su SF, Han F, Zhao C, Chen CY, Xiao WW, Li JX, Lu TX: Long-term outcomes of early-stage nasopharyngeal carcinoma patients treated with intensity-modulated radiotherapy alone. Int J Radiat Oncol Biol Phys 2012, 82(1):327-333.

5. Ma BB, Kam MK, Leung SF, Hui EP, King AD, Chan SL, Mo F, Loong H, Yu BK, Ahuja A, Chan AT: A phase II study of concurrent cetuximab-cisplatin and intensity-modulated radiotherapy in locoregionally advanced nasopharyngeal carcinoma. Ann Oncol 2012, 23(5):1287-1292.

6. Zhao LN, Zhou B, Shi M, Wang JH, Xiao F, Xu M, Luo SQ, Xue Y, Li JP, Tan $\mathrm{LN}$ : Clinical outcome for nasopharyngeal carcinoma with predominantly WHO II histology treated with intensity-modulated radiation therapy in non-endemic region of China. Oral Oncol 2012, 48(9):864-869.

7. Xiao WW, Huang SM, Han F, Wu SX, Lu LX, Lin CG, Deng XW, Lu TX, Cui NJ, Zhao C: Local control, survival, and late toxicities of locally advanced nasopharyngeal carcinoma treated by simultaneous modulated accelerated radiotherapy combined with cisplatin concurrent chemotherapy: long-term results of a phase 2 study. Cancer 2011, 117(9):1874-1883.

8. Lai SZ, Li WF, Chen L, Luo W, Chen YY, Liu LZ, Sun Y, Lin AH, Liu MZ, Ma J: How does intensity-modulated radiotherapy versus conventional twodimensional radiotherapy influence the treatment results in nasopharyngeal carcinoma patients? Int J Radiat Oncol Biol Phys 2011, 80(3):661-668.

9. Wong FC, Ng AW, Lee VH, Lui CM, Yuen KK, Sze WK, Leung TW, Tung SY: Whole-field simultaneous integrated-boost intensity-modulated radiotherapy for patients with nasopharyngeal carcinoma. Int J Radiat Oncol Biol Phys 2010, 76(1):138-145.

10. Tham IW, Hee SW, Yeo RM, Salleh PB, Lee J, Tan TW, Fong KW, Chua ET, Wee JT: Treatment of nasopharyngeal carcinoma using intensity- modulated radiotherapy-the national cancer centre singapore experience. Int J Radiat Oncol Biol Phys 2009, 75(5):1481-1486.

11. Wolden SL, Chen WC, Pfister DG, Kraus DH, Berry SL, Zelefsky MJ: Intensitymodulated radiation therapy (IMRT) for nasopharynx cancer: update of the Memorial Sloan-Kettering experience. Int J Radiat Oncol Biol Phys 2006, 64(1):57-62.

12. Kwong DL, Sham JS, Leung LH, Cheng AC, Ng WM, Kwong PW, Lui WM, Yau CC, Wu PM, Wei W, Au G: Preliminary results of radiation dose escalation for locally advanced nasopharyngeal carcinoma. Int J Radiat Oncol Biol Phys 2006, 64(2):374-381.

13. Kam MK, Teo PM, Chau RM, Cheung KY, Choi PH, Kwan WH, Leung SF, Zee B, Chan AT: Treatment of nasopharyngeal carcinoma with intensitymodulated radiotherapy: the Hong Kong experience. Int J Radiat Oncol Biol Phys 2004, 60(5):1440-1450.

14. Liu MT, Hsieh CY, Chang TH, Lin JP, Huang CC, Wang AY: Prognostic factors affecting the outcome of nasopharyngeal carcinoma. Jpn J Clin Oncol 2003, 33(10):501-508.

15. Lee N, Xia P, Quivey JM, Sultanem K, Poon I, Akazawa C, Akazawa P, Weinberg $V$, Fu KK: Intensity-modulated radiotherapy in the treatment of nasopharyngeal carcinoma: an update of the UCSF experience. Int J Radiat Oncol Biol Phys 2002, 53(1):12-22.

16. Kwong DL, Pow EH, Sham JS, McMillan AS, Leung LH, Leung WK, Chua DT, Cheng AC, Wu PM, Au GK: Intensity-modulated radiotherapy for early-stage nasopharyngeal carcinoma: a prospective study on disease control and preservation of salivary function. Cancer 2004, 101(7):1584-1593.

17. Dawson LA, Anzai Y, Marsh L, Martel MK, Paulino A, Ship JA, Eisbruch A: Patterns of local-regional recurrence following parotid-sparing conformal and segmental intensity-modulated radiotherapy for head and neck cancer. Int J Radiat Oncol Biol Phys 2000, 46(5):1117-1126.

18. Orlandi E, Tomatis S, Potepan P, Bossi P, Mongioj V, Carrara M, Palazzi M, Franceschini M, Bergamini C, Locati L, lannacone E, Guzzo M, Ibba T, Crippa F, Licitra L, Pignoli E, Fallai C: Critical analysis of locoregional failures following intensity-modulated radiotherapy for nasopharyngeal carcinoma. Future Oncol 2013, 9(1):103-114.

19. Cannon DM, Lee NY: Recurrence in region of spared parotid gland after definitive intensity-modulated radiotherapy for head and neck cancer. Int J Radiat Oncol Biol Phys 2008, 70(3):660-665.

20. ICRU Report 50: Prescribing, Recording, and Reporting Photon Beam Therapy. Bethesda, MD: International Commission on Radiation Units and Measurements; 1993.

21. ICRU Report 62: Prescribing, Recording, and Reporting Photon Beam Therapy (Supplement to ICRU Report 50). Bethesda, MD: International Commission on Radiation Units and Measurements; 1999.

22. Ho FC, Tham IW, Earnest A, Lee KM, Lu JJ: Patterns of regional lymph node metastasis of nasopharyngeal carcinoma: a meta-analysis of clinical evidence. BMC Cancer 2012, 12:98.

23. Hong B, Lui WW, Hashiguchi M, Hui EP, Chan AT: Targeting tumor hypoxia in nasopharyngeal carcinoma. Head Neck 2013, 35(1):133-145.

24. Lin S, Pan J, Han L, Zhang X, Liao X, Lu JJ: Nasopharyngeal carcinoma treated with reduced-volume intensity-modulated radiation therapy: report on the 3-year outcome of a prospective series. Int J Radiat Oncol Biol Phys 2009, 75(4):1071-1078.

\section{doi:10.1186/1748-717X-9-60}

Cite this article as: Kong et al:: Patterns of local-regional failure after primary intensity modulated radiotherapy for nasopharyngeal carcinoma. Radiation Oncology 2014 9:60. 